\title{
Scaling of the critical current in ITER type niobium-tin superconductors in relation to the applied field, temperature and uni-axial applied strain
}

\author{
Arno Godeke, Bennie ten Haken and Herman H.J. ten Kate \\ University of Twente, Faculty of Applied Physics, P.O. Box 217, 7500 AE Enschede, The Netherlands
}

\begin{abstract}
The three dimensional surface of the critical current density versus field and temperature $J_{c}(B, T)$ of niobiumtin is a function of the strain state of the superconductor. $A$ brief review of literature on this subject is presented. The $J_{c}(B)$ function is described by the relations for flux pinning. The temperature and strain dependencies are added to this relation. This results in a unifying scaling law for A15 materials, which is verified for different niobium-tin conductors with respect to all the relevant variables, i.e. field, temperature and uni-axial strain. $\mathrm{Nb}_{3} \mathrm{Sn}$ conductors from 9 manufacturers are measured in the frame work of the third ITER benchmark tests on critical current*. The investigated ranges are: applied field from 7 to $13 \mathrm{~T}$, temperature from 4.2 to $8 \mathrm{~K}$ and applied strain from -0.4 to $+0.8 \%$. Special attention is paid to the region of compressive axial strain, which is the most relevant state of strain for superconductors under thermal compression in practical applications.
\end{abstract}

\section{INTRODUCTION}

A large amount of conductors is characterized in the frame of the third ITER benchmark tests. The critical current of the conductors is measured as a function of the applied field, temperature and uni-axial strain. The data are used to verify an improved scaling law, which is deduced from scaling relations available through literature and a comparison is made between two different descriptions for the axial strain dependency in A15 materials. The scaling relation is expected to be an accurate description for the $J_{c}(B, T, \varepsilon)$ dependency of $\mathrm{Nb}_{3} \mathrm{Sn}$ conductors, in the compressive axial strain regime.

\section{SCALING RELATIONS}

\section{A. Field dependence}

The description of the critical current density of type II superconductors is based on the critical state concept [1,2], in which at the critical current density $J_{c}$, the Lorentz force is exactly counteracted by the maximum pinning force $F_{p}$. In that case, the critical state is represented by [3]:

$$
J_{c}(B) \times B=-F_{p}(B) .
$$

Various pinning models $[4,5]$ give a relationship for the pinning force and the applied field:

$$
F_{p}(b)=C \cdot b^{p} \cdot(1-b)^{q}=C \cdot f(b),
$$

which is valid for $0.3<<b<0.9$ and over a wide temperature range. In this relation is $b=B / B_{c 2}$ and the function $f$ is implicitly defined. The parameter $C$ is a scaling constant for the maximum pinning force, which is proportional to the critical current. For $\mathrm{Nb}_{3} \mathrm{Sn} p \approx 0.5$ and $q \approx 2$ appear to be appropriate values.

Manuscript received September 13, 1998

*These investigations are part of the NET contracts No. 94-338 and 96-434 between the European Union and the University of Twente.

\section{B. Temperature dependence}

Fietz and Webb [6] state that the temperature dependence of the pinning force can be written as a function of a temperature dependent $B_{c 2}$, according to:

$$
F_{p}(B, T)=C \cdot \kappa(T)^{-\gamma} \cdot B_{c 2}(T)^{v} \cdot f\left(B / B_{c 2}(T)\right),
$$

with $2 \leq v \leq 3$, but comes closest to 2 [5,7]. The function $\kappa(T)^{-\gamma}$ is introduced in order to insert qualitatively a temperature dependent $\kappa$, with $1<\gamma<3$. A few years earlier they showed [8] that, neglecting the temperature dependence of the GinzburgLandau parameter, the temperature dependence of $B_{c 2}(T)$ is within $10 \%$ proportional to $\left(1-t^{2}\right)$ for $T \ll<T_{c}$, with $t=T / T_{c}$.

A refinement is made by Summers et al. [9], who empirically fitted the temperature dependence of the Ginzburg-Landau parameter to:

$$
K(t)=\kappa(t) / \kappa(0)=1-0.31 t^{2} \cdot(1-1.77 \ln t),
$$

and introduced it into the temperature dependence of $B_{c 2}$ via:

$$
\beta(t)=B_{c 2}(t) / B_{c 2}(0)=\left(1-t^{2}\right) \cdot K(t) .
$$

Note that the influence of this correction on the pinning force, depends on the relation between the two powers $v$ and $\gamma$. In the case that $v=2$ and $\gamma=2$, the influence of $\kappa(T)$ on $F_{p}(B, T)$ disappears.

The exact shape of $\kappa(T)$ is strongly material dependent, as shown by Fietz and Webb [8] for different NbTi alloys. It is reasonable to assume also a strong material dependence for $\mathrm{Nb}_{3} \mathrm{Sn}$. This inherently means that a certain error in the model for $J_{c}(B, T)$ can arise from an inaccurate description of $B_{c 2}(T)$ and/or a not well known dependency of $\kappa(T)$.

\section{Strain dependence}

Ekin [10] stated that the influence of a mechanical deformation on the pinning force should be written in the same explicit way as the temperature dependency:

$$
F_{p}(B, \varepsilon)=C \cdot B_{c 2}(\varepsilon)^{n} \cdot f\left(B / B_{c 2}(\varepsilon)\right),
$$

with $n=1 \pm 0.3$ for a measurement at a reference temperature (e.g. $4.2 \mathrm{~K}$ ). The introduced strain dependence function $S(\varepsilon)=B_{c 2}(4.2, \varepsilon) / B_{c 2 \mathrm{~m}}(4.2)$ is an empirical fit of the $B_{c 2}(\varepsilon)$ data at $4.2 \mathrm{~K}$, scaled to the maximum $\left(B_{c 2 m}\right)$ in the strain dependency curve.

For a combination of (3) and (6) it is necessary to scale also $T_{c}$ with strain. The strain dependence of $T_{c}$, at $B=0 \mathrm{~T}$ is written as a function of the strain dependence of $B_{c 2}$ at $4.2 \mathrm{~K}$ according to $[10,11]$ :

$$
T_{c}(\varepsilon) / T_{c m}=\left(B_{c 2}(4.2, \varepsilon) / B_{c 2 m}(4.2)\right)^{1 / w},
$$

where $w \approx 3$ for A15 materials and $T_{c m}$ is the critical temperature at zero applied field at the maximum in the strain curve. 
Combining (1), (3) and (6), under the condition that $S(\varepsilon)$ is independent of temperature leads to:

$$
\begin{aligned}
& J_{c}(B, T, \varepsilon)=\frac{C \cdot \beta(T, \varepsilon)^{v}}{B \cdot K(T, \varepsilon)^{\gamma}} \cdot S(\varepsilon)^{n} \cdot f\left(B / B_{c 2}(T, \varepsilon)\right), \\
& \text { with: } \quad T_{c}(\varepsilon)=T_{c m} \cdot S(\varepsilon)^{1 / w} \\
& K(T, \varepsilon)=1-0.31 \cdot\left(T / T_{c}(\varepsilon)\right)^{2} \cdot\left(1-1.77 \cdot \ln \left(T / T_{c}(\varepsilon)\right)\right) \\
& \beta(T, \varepsilon)=\left(1-\left(T / T_{c}(\varepsilon)\right)^{2}\right) \cdot K(T, \varepsilon) \\
& \text { and } B_{c 2}(T, \varepsilon)=B_{c 2 m}(0) \cdot S(\varepsilon) \cdot \beta(T, \varepsilon)
\end{aligned}
$$

The critical current at a certain temperature $T$, applied field $B$ and applied strain $\varepsilon$, is then written as a function of three material parameters $T_{c m}(0 \mathrm{~T}), B_{c 2 m}(0 \mathrm{~K})$ and $C$, and the strain dependency function $S(\varepsilon)$. The strain $\varepsilon$ in the above formulas is an effective value, representing the intrinsic state of strain that is present in the superconductor. The assumption that $S(\varepsilon)$ is independent of temperature seems reasonable, considering the fact that (3) holds for different samples, so inherently for different strain states.

An empirical axial strain dependence function $S\left(\varepsilon_{a}\right)$ is given by the power law of Ekin [10]:

$$
S\left(\varepsilon_{a}\right)=1-a \cdot\left|\varepsilon_{a}-\varepsilon_{m}\right|^{u},
$$

where $\varepsilon_{m}$ is equal to the applied axial strain at which the maximum in $J_{c}$ occurs. This description has proven to work very well for axially elongated wires, if two different values for the strain scaling constant $a$ are used. Typical values for $\mathrm{Nb}_{3} \mathrm{Sn}$ are $a \approx 900$ for $\varepsilon_{a}<\varepsilon_{m}, a \approx 1250$ for $\varepsilon_{a}>\varepsilon_{m}$ and $u=1.7$.

Alternatively, $S(\varepsilon)$ can be written as a special (axial) form of a three dimensional deviatoric strain model, that utilizes constant overall Poisson ratios in the composite conductor. The strain function for axially deformed conductors, in which the upper critical field is normalized to the maximum in the strain dependency curve, then exhibits the following non-hydrostatic strain dependency $[12,13,14]$ :

$$
S\left(\varepsilon_{a}\right)=\frac{1-C_{a} \cdot \sqrt{\left(\varepsilon_{a}+\delta\right)^{2}+\left(\varepsilon_{0, a}\right)^{2}}}{1-C_{a} \cdot \varepsilon_{0, a}},
$$

which uses a scaling constant $C_{a}$ that is defined slightly different than in previous publications $[12,14]$. The factor $\varepsilon_{0, a}$ includes the remaining strain state of the superconductor at the maximum in the strain dependency curve and is found to be relatively small $(<0.2 \%) . \delta$ is determined by the thermally induced pre-strain. Note that (14) does not account for non-elastic deformations with a changing Poisson ratio (e.g. yielding or cracking).

\section{MEASUREMENTS}

\section{A. Sample material}

In order to verify the scaling law, the data from a large number of measurements are analyzed. All samples from one producer are reacted together under vacuum conditions, in order to treat the various samples as equally as possible. A selected
TABLE 1:

SUMMARY OF THE CONDUCTOR CHARACTERISATIONS.

\begin{tabular}{ccccccc}
\hline $\begin{array}{c}\text { Produ- } \\
\text { cer }\end{array}$ & Process & $\begin{array}{c}\mathrm{Cu} / \\
\text { non-Cu }\end{array}$ & $\begin{array}{c}\text { Diff. } \\
\text { Barr }\end{array}$ & $\begin{array}{c}\text { Tern. } \\
\text { Addit. }\end{array}$ & $\begin{array}{c}\text { RRR } \\
{[-]}\end{array}$ & $\begin{array}{c}\text { Spread } \\
\text { in } I_{c}[\%]\end{array}$ \\
\hline A & Bronze & 1.49 & $\mathrm{Ta}$ & - & 147 & \pm 0.5 \\
$\mathrm{~B}$ & Int. tin & 1.38 & $\mathrm{Nb} / \mathrm{Ta}$ & $1 \% \mathrm{Ti}$ & 80 & \pm 0.9 \\
$\mathrm{C}$ & Int. tin & 1.59 & $\mathrm{Ta}$ & - & 130 & \pm 0.3 \\
$\mathrm{D}$ & Bronze & 1.49 & $\mathrm{Ta}$ & $7.5 \% \mathrm{Ta}$ & 150 & \pm 0.8 \\
$\mathrm{E}$ & Int. tin & 1.84 & $\mathrm{Ta}$ & - & 144 & \pm 16 \\
$\mathrm{~F}$ & Int. tin & 1.61 & $\mathrm{Nb} / \mathrm{Ta}$ & $1 \% \mathrm{Ti}$ & 213 & \pm 16 \\
\hline
\end{tabular}

overview of the conductor specifications, partly deducted from the characterizations is given in Table 1.

\section{B. Deformation experiments}

The set-up to measure the critical current as a function of field, temperature and axially applied strain is described elsewhere $[13,15]$. The samples are heat-treated on a stainless steel holder and then transferred to a brass substrate. The wires are tightly soldered to the substrate with $\mathrm{SnAg}$ solder, in order to get a well defined and reproducible pre-strain. Voltage-current measurements are taken as a function of applied axial strain at applied field values of 10 and $13 \mathrm{~T}$ at liquid Helium bath temperature (atmospheric pressure) and $6.5 \mathrm{~K}$. Due to the limited sample length, the voltage is measured across a length of only $5 \mathrm{~mm}$ and the critical currents are determined at a voltage level of $5 \cdot 10^{-5} \mathrm{~V} / \mathrm{m}$. This is relatively high compared to the $I_{c}$-measurements on the Ti-6Al-4V holder, but low compared to the "standard" level of $10^{-4} \mathrm{~V} / \mathrm{m}$.

\section{Critical current measurements}

The $I_{c}$-measurements as a function of the applied field and temperature are performed on a Ti-6Al-4V holder. After heat treatment on the holder, the samples are fixed with epoxy resin, in order to get a well defined, and reproducible pre-strain condition. A more detailed description of the set-up is given in [13]. Voltages are measured across a sample length of $50 \mathrm{~cm}$ and the $I_{c}$-values are determined at a voltage criterion of $10^{-5} \mathrm{~V} / \mathrm{m}$.

The $I_{c}(B)$ measurements are performed at liquid Helium temperature for field values ranging from 7 to $13 \mathrm{~T}$. From each producer, four to six samples are measured at $12 \mathrm{~T}$ and $4.2 \mathrm{~K}$, in order to get an indication of the spread in critical current per sample type (see Table 1) and one sample is selected on which to perform the field dependency measurements.

The $I_{c}(T)$ measurements are performed at a background field of $13 \mathrm{~T}$, for temperatures ranging from 5 to $8 \mathrm{~K}$. For producers $\mathrm{A}, \mathrm{D}$ and $\mathrm{F}$, the same samples are used as in the $I_{c}(B)$ characterization. For producers $\mathrm{B}, \mathrm{C}$ and $\mathrm{E}$, different samples are used. In order to compare the $I_{c}(B, T)$ characterization with the deformation experiments, the V-I transitions are extrapolated to a voltage criterion of $5 \cdot 10^{-5} \mathrm{~V} / \mathrm{m}$. This results in an extrapolation error in the $I_{c}$ which is smaller than $1 \%$, except for conductor $\mathrm{D}$, where a non-constant slope of the transition results in an extrapolation error estimated at $3 \%$.

\section{VERIFICATION}

In order to verify the validity of (8), its parameters are determined to describe all the available data. A separation is made between parameters, which are considered to be properties 
TABLE 2:

MATERIAL PARAMETERS FOR $\mathrm{NB}_{3} \mathrm{SN}$

\begin{tabular}{lcccccccc}
\hline Parameter & $n$ & $p$ & $q$ & $v$ & $\gamma$ & $w$ \\
\hline Value & 1 & 0.5 & 2 & 2 & 1 & 3 \\
\hline \multicolumn{8}{c}{ TABLE 3: } \\
\multicolumn{8}{c}{ CONDUCTOR PARAMETERS } \\
\hline Prod & $I_{C u}$ & $\delta_{T i}$ & $\delta_{\text {Brass }}$ & $\varepsilon_{0, a}$ & $C_{a}$ & $B_{c 2 m}{ }^{*}(0)$ & $T_{c m}{ }^{*}(0)$ \\
& {$[\mathrm{A}]$} & {$[\%]$} & {$[\%]$} & {$[\%]$} & & {$[\mathrm{T}]$} & {$[\mathrm{K}]$} \\
\hline A & 1.67 & -0.22 & -0.51 & 0.12 & 38.0 & 33.3 & 17.8 \\
B & 0.79 & -0.21 & -0.60 & 0.14 & 37.4 & 31.2 & 17.0 \\
C & 1.37 & -0.10 & -0.62 & 0.26 & 41.5 & 29.0 & 16.9 \\
D & 1.60 & -0.14 & -0.59 & 0.08 & 37.4 & 32.2 & 17.4 \\
E & 1.45 & -0.11 & -0.63 & 0.19 & 40.8 & 27.8 & 18.1 \\
F & 2.31 & -0.02 & -0.43 & 0.17 & 39.1 & 28.9 & 16.7 \\
\hline
\end{tabular}

of $\mathrm{Nb}_{3} \mathrm{Sn}$ (Table 2) and parameters, which depend on the production process and preparation method (Table 3). Since all samples of one producer are reacted at once, they should inhibit the same $B_{c 2 m}$ and $T_{c m}$ and only parameter $C$, the overall scaling constant for the critical current, is allowed to change per sample.

The latter however, is only necessary for the samples $\mathrm{D}$, which obtain a larger inaccuracy in the $I_{c}$ determination due to the extrapolation to the $5 \cdot 10^{-5} \mathrm{~V} / \mathrm{m}$ level, caused by a much lower n-value $(\sim 15)$ compared to the other samples $(-35)$ [16], and for samples $\mathrm{E}$ and $\mathrm{F}$, that have a relatively large variation in critical current, even on the Ti-6Al-4V holder (see Table 1).

Two pre-strain values are defined. One for the experiments on the Ti-6Al-4V holder $\left(\delta_{T i}\right)$ and one for the deformation experiments on the brass substrate $\left(\delta_{\text {Brass }}\right)$. A correction is made for the current that is running through the material parallel to the $\mathrm{Nb}_{3} \mathrm{Sn}$ at the selected voltage criterion. This normal current $\left(I_{C u}\right)$ is determined at $20 \mathrm{~K}$ and $B=0 \mathrm{~T}$ and subtracted from the measured $I_{c}$ values.

First, the two possible definitions of $S(\varepsilon)$ are compared with the samples of manufacturer $A$ because of the extremely good reproduction of the $I_{c}(B, T, \varepsilon)$ data on all different samples. The measured data for samples A1 (open) and A2 (filled) are shown in Figure 1. The points represent the measured data. The dashed line represents scaling according to (13), across the complete strain range ( $a=883$ for $\varepsilon_{a}<\varepsilon_{m}, a=4376$ for $\varepsilon_{a}>\varepsilon_{m}$ and $u=1.7)$ and the dotted line (13) with the negative applied strain values omitted ( $a=1040$ for $\varepsilon_{a}<\varepsilon_{m}, a=4096$ for $\varepsilon_{a}>\varepsilon_{m}$ and $u=1.7)$. The latter shows an improved accuracy for higher strain

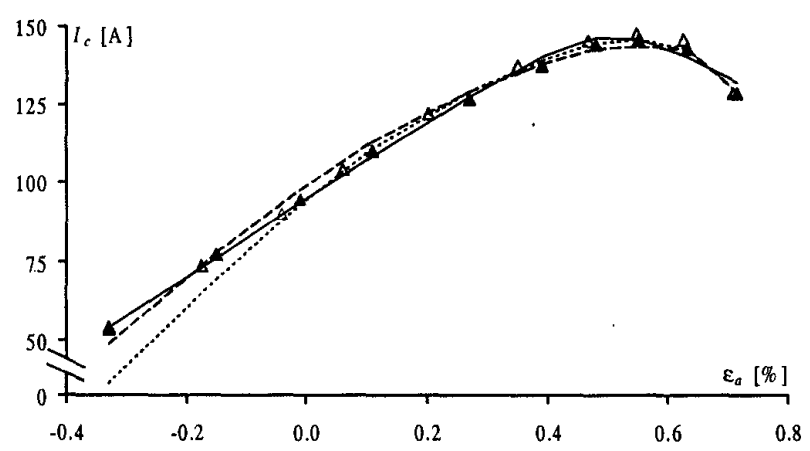

Figure 1: The critical current versus applied strain for conductor A, at $B=10 \mathrm{~T}$ and $T=6.5 \mathrm{~K}$. The other combinations of field and temperature show similar behavior. The lines are described in the text.

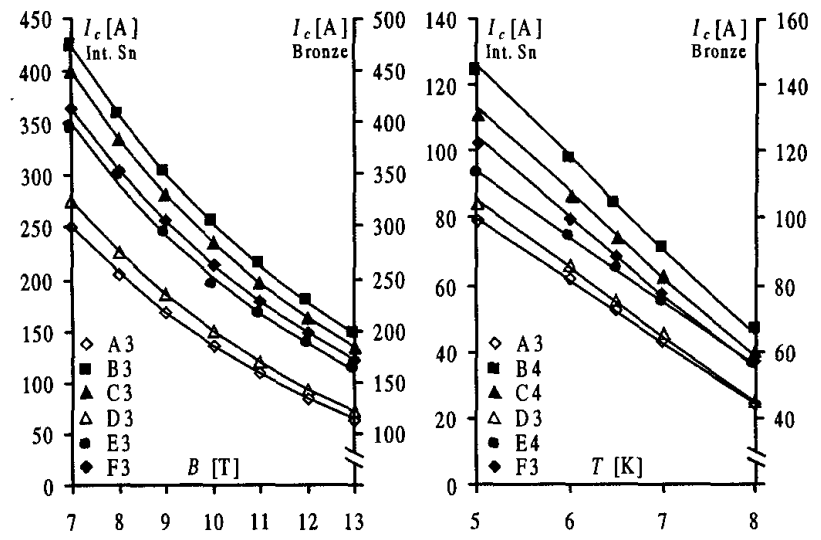

Figure 2: The $I_{c}(B)$ data at liquid Helium temperature and $I_{c}(T)$ data at $13 \mathrm{~T}$ for all manufacturers. The points are measured and the lines are calculated with (8), with parameters as listed in Table 2 and Table 3. The strain state is calculated with $\varepsilon_{a}=0$ and $\delta=\delta_{\pi i}$. The current axis for the bronze conductors (open markers) is shifted for clarity reasons.

values. The continuous line is calculated with (14), across the entire strain range, with parameters as given in Table 2 and Table 3. In particular the regime from -0.4 to $+0.4 \%$ applied strain is better described with (14). Since this is the most relevant regime for practical applications, this equation is chosen for the remaining analysis.

The critical current data of all samples, as measured on the Ti-6Al-4V holder, are given in Figure 2. It is clear that the field and temperature descriptions in (8) work very well with one set of parameters (Table 2 and Table 3 ) across all the samples from one producer, although a small improvement can be made on the temperature dependence. This is confirmed by earlier experiments on conductors $\mathrm{B}$ and $\mathrm{D}$, in which a strong deviation occurs at higher temperatures [17].

The deformation experiments on conductor $\mathrm{A}$ are presented in Figure 3. The good correlation between all the $I_{c}$ values determined on conductor $\mathrm{A}$ is an important indication for the validity of the presented description for $J_{c}(B, T, \varepsilon)$. The fact that the current amplitude correlates so well with a single value for parameter $C$ is an important verification for the experimental procedures and in particular the sample preparation. An important limitation of the considered description is the $I_{c}$ strain dependence around the maximum in $I_{c}(\varepsilon)$. The small asymmetry

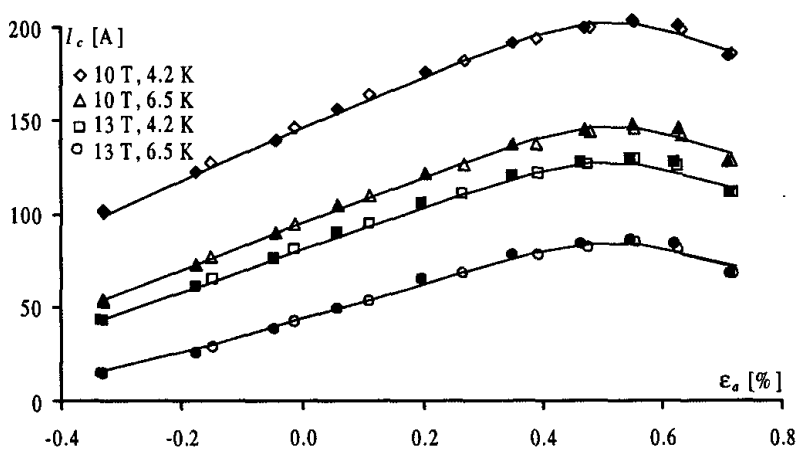

Figure 3: The deformation measurements for conductors A1 and A2 (open and filled markers). The points are measured and the lines are calculated with (8), with parameters as listed in Table 2 and Table 3. 


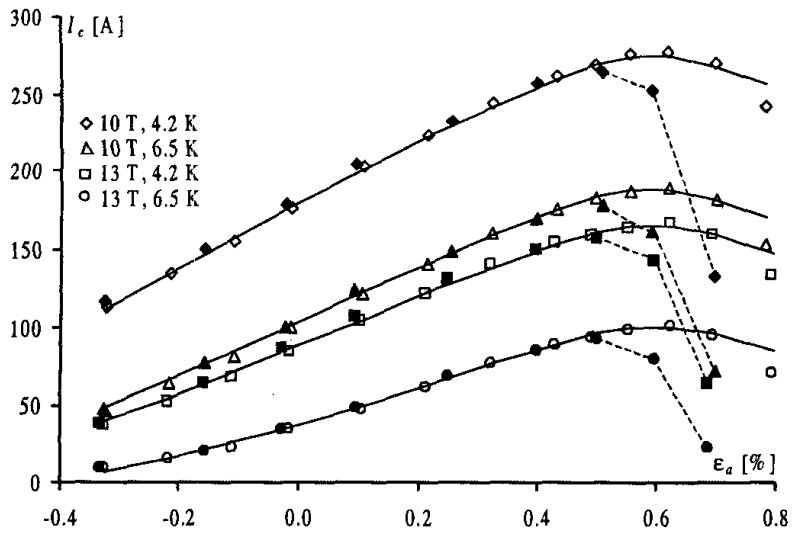

Figure 4: The deformation measurements for conductors B1 and B2 (open and filled markers). The points are measured and the solid lines are calculated with (8), with parameters as listed in Table 2 and Table 3 . The dashed lines indicated the deviation from (8).

that is observed in the $I_{c}$-strain dependence cannot be covered by this semi-elastic description for the deviatoric strain dependence.

The deformation experiments on conductor $\mathrm{B}$ are given in Figure 4. The only deviation in the $I_{c}$ measurements occurs at large tensile strain for sample B2, where an increased reduction is observed. An explanation for this sudden reduction is the occurrence of non-elastic deformations like yielding or cracking in the conductor that has a highly stressed matrix at this point.

Another important conclusion that can be drawn from this measurement, as can be seen in Figure 4, is that normalized scaling of $I_{c}$ with respect to the maximum in the strain curve, will lead to irreproducibility's in the $I_{c}, T_{c}$ and $B_{c 2}$, across different samples of a single manufacturer in the compressive

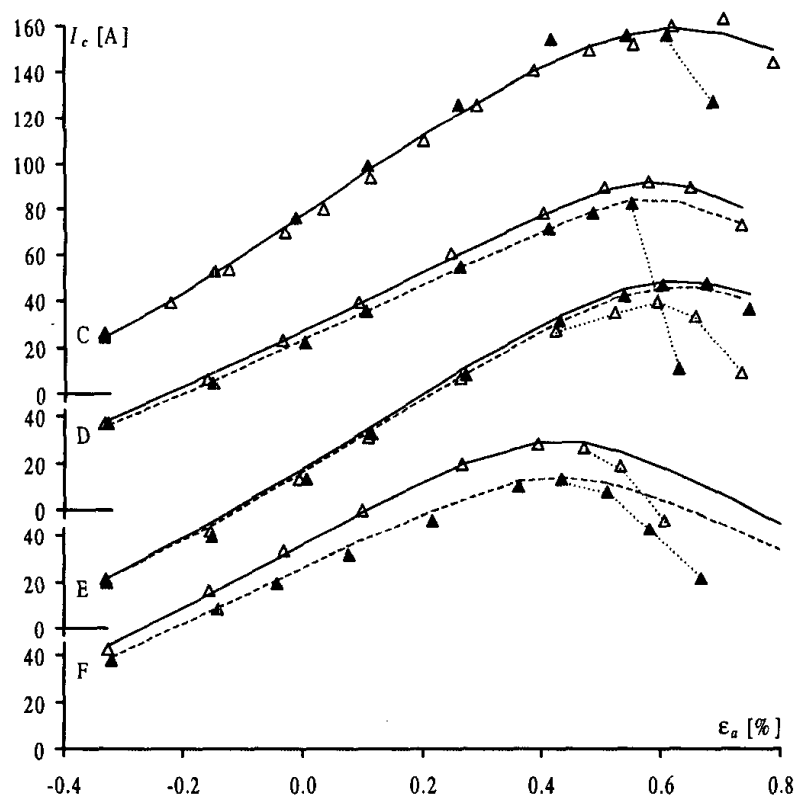

Figure 5: The deformation experiments on the conductors $\mathrm{C}-\mathrm{F}$ at $B=10 \mathrm{~T}$ and $T=6.5 \mathrm{~K}$. Open or filled markers indicate different samples. The solid and dashed lines are calculated with (8), with parameters as listed in Table 2 and Table 3 . The solid lines are calculated with a different value for parameter $C$ than the dashed lines. The dotted lines indicate deviations from (8). strain regime. This is caused by a different interpretation of the strain axis.

The $I_{c}\left(B, T, \varepsilon_{a}\right)$ data, combined with the description according to (8) of the remaining samples is given in Figure 5, for $B=10 \mathrm{~T}$ and $T=6.5 \mathrm{~K}$. The other field and temperature combinations show a similar behavior. The deviations can be correlated to non-elastic deformations, similar to the data for sample B2. For samples $\mathrm{F}$, the description for $J_{c}(B, T, \varepsilon)$ is only accurate in the compressive regime, where the matrix deformation is small.

\section{CONCLUSIONS}

1. An improved scaling law for $J_{c}(B, T, \varepsilon)$ is deduced from relations, which are available in literature. The model is verified with experimental results and delivers an accurate description of the three dimensional critical surface of $\mathrm{Nb}_{3} \mathrm{Sn}$ as a function of the strain state, in particular in the compressive axial strain regime. The relation makes it possible to describe the behavior of all samples of one type of conductor with a single set of parameters.

2. The highly stressed region around the maximum in the strain dependency curve cannot always be covered by the linear, axial approximation of the deviatoric strain model (14). This part is better covered with the asymmetric power law description (13). For technical applications with a large axial compression, like CIC conductors and magnet systems, (14) will be preferable. 3. A future improvement can be made in the deviatoric strain description, to allow for the asymmetry in the strain dependency curve via non-linear mechanical behavior, like plastic deformation. This will extend its validity range in the tensile strain region, but will not be able to cover the variation in $I_{c}(\varepsilon)$, that is observed between the samples of some conductors.

4. A present constraint for the scaling relation is the limited verification of the temperature dependency part. An important improvement will be an extension to temperatures close to $T_{c}$.

\section{REFERENCES}

[1] C.P. Bean, Phys. Rev. Lett., Vol. 8, p. 250, 1962

[2] Y.B. Kim, C.F. Hempstead and A.R. Strnad, Phys. Rev., Vol. 129, p. $528,1963$.

[3] J.E. Evetts, A.M. Campbell and D. Dew-Hughes, J. Phys. C., Vol. 1, p. 715,1968

[4] E.J. Kramer, J. Appl. Phys., Vol. 44, p. 1360, 1973.

[5] D. Dew-Hughes, Phil. Mag., Vol. 30, p. 293, 1974

[6] W.A. Fietz and W.W. Webb, Phys. Rev., Vol. 178-2, p. 657, 1969.

[7] D.P. Hampshire, H. Jones and E.W.J. Mitchell, IEEE Trans. Mag., Vol. 21, p. 289, 1985.

[8] W.A. Fietz and W.W. Webb, Phys. Rev., Vol 161-2, p. 423, 1967.

[9] L.T. Summers, M.W. Guinan, J.R. Miller and P.A. Hahn, IEEE Trans. Magn., Vol 27-2, p. 2041, 1991.

[10] J.W. Ekin, Cryogenics, Vol. 20, p. 611, 1980.

[11] D.O. Welch, Adv. in Cryo. Eng., Vol. 26, p. 48, 1980.

[12] B. ten Haken, A. Godeke and H.H.J. ten Kate, IEEE Trans. on Supercond., Vol. 5(2), p. 1909, 1995.

[13] B. ten Haken, A. Godeke and H.H.J. ten Kate, submitted to Journal of Applied Physics.

[14] B. ten Haken, H.H.J. ten Kate, T. Takeuchi and H. Wada, Adv, in Cryo. Eng., Vol. 44, p. 991, 1998.

[15] B. ten Haken, A. Godeke and H.H.J. ten Kate, Proc. MT-15, Edited by L. Liangzhen et. al., p. 985, 1998.

[16] H.G. Knoopers, A. Nijhuis, E.J.G. Krooshoop, H.H.J. ten Kate, P. Bruzzone, P.J. Lee and A.A. Squitieri, presented at EUCAS-97, Veldhoven, The Netherlands, 1997.

[17] A. Martinez and J.L. Duchateau, Cryogenics, Vol. 37, p. 865, 1997. 